\title{
The Need for Change: Understanding Emotion Regulation Antecedents and Consequences using Ecological Momentary Assessment
}

\author{
Desirée Colombo ${ }^{1}$, Javier Fernandez Alvarez ${ }^{2}$, Carlos Suso-Ribera ${ }^{1}$, Pietro Cipresso ${ }^{2,3}$, Hristo Valev ${ }^{4,5}$, Tim \\ Leufkens $^{4}$, Corina Sas ${ }^{5}$, Azucena Garcia Palacios ${ }^{1,6}$, Giuseppe Riva ${ }^{2,3}$, Cristina Botella ${ }^{1,6}$
}

\author{
${ }^{1}$ Department of Basic Psychology, Clinic and Psychobiology, Universitat Jaume I, Spain \\ ${ }^{2}$ Department of Psychology, Università Cattolica del Sacro Cuore, Italy \\ ${ }^{3}$ Applied Technology for Neuro-Psychology Lab, IRCCS Istituto Auxologico Italiano, Italy \\ ${ }^{4}$ Philips Research, High Tech Campus, Eindhoven, Netherlands \\ ${ }^{5}$ School of Computing and Communications, Lancaster University, UK \\ ${ }^{6}$ CIBER Fisiopatología Obesidad y Nutrición (CIBERobn), Instituto Salud Carlos III, Spain
}

\begin{abstract}
In recent decades, emotion regulation (ER) has been one of the most widely studied constructs within the psychological field. Nevertheless, laboratory experiments and retrospective assessments have been the two most common strands of ER research, thus leaving open several crucial questions about ER antecedents and consequences in daily life. Beyond traditional methods, ecological momentary assessment (EMA) has the potential to capture ER dynamics during the flow of daily experiences, in real-life settings and through repeated measurements. Here, we discuss what we currently know about ER antecedents and consequences. We will compare findings from previous literature to findings from EMA studies, pointing out both similarities and differences, as well as questions that can be answered better with the EMA approach.
\end{abstract}

Keywords: Emotion Regulation, Ecological Momentary Assessment, Emotion Regulation Antecedents, Emotion Regulation Consequences. 


\section{Introduction}

Emotion regulation (ER) is a process that every individual explicitly or implicitly implements in order to downregulate or upregulate emotional states and achieve personal goals (Gross, 2015). ER can be conceptualized as a complex process with physiological underpinnings, behavioral responses, and affective and cognitive correlates. Adding to this complexity, the preponderant role played by contextual and momentary factors is increasingly emerging, suggesting the dynamic and situational nature of this process (Aldao, 2013). To date, research into ER has mostly been based on laboratory experiments, thus leaving situated and momentary aspects relatively understudied. Additionally, most studies have relied on retrospective questionnaires, that consider ER to be a trait (i.e. a relatively stable and cross-situational tendency of an individual). However, trait measures only modestly correlate with ER in daily life (Brockman, Ciarrochi, Parker, \& Kashdan, 2017), which supports the need for a more ecological approach to the investigation of this process.

An alternative strategy to laboratory experiments and retrospective assessments can be found in ecological momentary assessment (EMA), a procedure that makes it possible to collect repeated inputs of thoughts, feelings and behaviors close in time to the experience and in real-life contexts. The past few years have seen a surge in studies using EMA due to the increased availability of mobile applications (Colombo, Fernandez Alvarez, Palacios, et al., 2019) for both subjective (Suso-Ribera et al., 2018) and objective (Marzano et al., 2015) data collection, and this method has already significantly increased our knowledge about ER outside laboratory settings (Bylsma \& Rottenberg, 2011). In fact, a more complex representation of the way people regulate emotions is emerging that emphasizes the importance of capturing this process in the context where emotions are regulated.

In the present paper, we will provide an overview of the available literature on ER antecedents and consequences. To do so, we will relate EMA findings to the broader literature based on laboratory and retrospective studies, pointing out both similarities and differences, as well as questions that can be better answered with the EMA approach. We will suggest EMA as a suitable methodology to extend our understanding of ER, and we will propose a technology-based model to concurrently explore the affective, physiological, and behavioral concomitants of ER.

\section{What are the antecedents of ER?}


Beyond individual differences (John \& Gross, 2007), ER can be understood as the result of a complex interplay among contextual and situational factors, momentary affect, and situation-specific goals.

\section{Contextual and situational factors}

Existing research has revealed the importance of the social context in ER, showing that both rapid changes in the social context (McRae, Heller, John, \& Gross, 2011) and stable contextual transactions (Srivastava, Tamir, McGonigal, John, \& Gross, 2009) influence this process. Additionally, context controllability has been shown to be critical for ER. Accordingly, Troy and colleagues demonstrated that higher reappraisal ability (i.e. the ability to down-regulate negative emotions) is associated with lower levels of depression after the exposure to an uncontrollable stressor, but it is associated with higher subsequent depression in the context of a controllable stressor (Troy, Shallcross, \& Mauss, 2013). Finally, the general context where the emotions are regulated can significantly impact ER. For example, one study shows that people are more likely to choose distraction in high-intensity negative situations, whereas in low-intensity negative situations people are more likely to adopt cognitive reappraisal, which involves rethinking the meaning of a stimulus in order to change its emotional impact (Sheppes, Scheibe, Suri, \& Gross, 2011).

EMA studies have confirmed the role of both the social context and context controllability in ER. Suppression, that is the inhibition of ongoing emotion-expressive behaviors, is more frequently adopted when other people are present in the environment (English, Lee, John, \& Gross, 2017) and it is used less frequently in situations where people feel high in social hierarchy (Catterson, Eldesouky, \& John, 2016). Additionally, individuals with higher well-being report greater reappraisal adoption in situations that are low in controllability (Haines et al., 2016). Beyond these results, EMA studies also extended the previous literature about contextual determinants of ER. The identification of the trigger for one's emotions has been shown to affect ER and, more specifically, to be tied to the use of certain strategies, such as reappraisal and savoring (i.e. the attempt to maintain and up-regulate positive emotions) (Heiy \& Cheavens, 2014). Furthermore, the type of emotion targeted also influences this process, so that people concurrently use more strategies in response to certain emotions, such as excitement and pride (Heiy \& Cheavens, 2014).

\section{Momentary affect}

According to the broaden-and-build theory, the experience of positive emotions fosters people's repertoires of thoughts and actions and encourages the development of new coping skills (Fredrickson, 2001). Evidence 
supporting this theory comes, for example, from a longitudinal study, showing that higher PA levels predict increased positive coping skills eight weeks later (Burns et al., 2008).

Nonetheless, most of the previous literature only relied on retrospective measurements of ER, which made it difficult to explore real-life relationships between momentary affect and ER. Fortunately, in recent years there has been a surge in research using the EMA approach for the exploration of affective antecedents. For example, high levels of momentary self-esteem, psychological adjustment, and PA have been found to trigger the use of cognitive reappraisal (Nezlek \& Kuppens, 2008). High levels of PA also predict greater adoption of problem solving (Pavani, Le Vigouroux, Kop, Congard, \& Dauvier, 2016) and mindfulness (Brockman et al., 2017), whereas momentary NA has been associated with greater use of suppression (Brockman et al., 2017) and rumination, which refers to the process of persistently thinking about one's feelings (Li, Starr, \& Hershenberg, 2017; Pavani et al., 2016). Interestingly, momentary affect also appears to influence the number of strategies adopted, so that the use of different concurrent ER strategies increases in the presence of high levels of momentary NA compared to PA (Brans, Koval, Verduyn, Lim, \& Kuppens, 2013).

\section{Situation-specific goals}

Along with the aforementioned contextual and affective factors, situation-specific goals have also been shown to influence ER. Overall, the previous literature has shown that individuals who frequently reappraise and distract tend to pursue prosocial and pro-hedonic goals more often, whereas people who habitually suppress tend to pursue contra-hedonic and impression management goals more frequently (Eldesouky \& English, 2018).

These results are consistent with the EMA literature, which has revealed that distraction and cognitive reappraisal are used more frequently when hedonic goals are pursued, whereas suppression is more likely to be implemented when achieving instrumental goals (English et al., 2017).

\section{What are the consequences of ER?}

In the previous section, we explained that ER can be understood as the outcome of a number of antecedents. However, ER also has multiple consequences on people's lives. In the following paragraphs, we will discuss two important outcomes that can be influenced by ER, namely affective and physiological states.

\section{Momentary affect}


A large amount of evidence from previous literature shows an influence of ER on subsequent affective states, and most of the research concludes that cognitive reappraisal has a more adaptive profile in terms of affective consequences, compared to suppression. Reappraisal is indeed related to experiencing less subsequent NA and more PA, whereas suppression leads to experiencing less PA and more NA (Gross \& John, 2003). Furthermore, frequent use of savoring has been associated with greater happiness and well-being (Quoidbach, Berry, Hansenne, \& Mikolajczak, 2010), whereas recurrent use of rumination (Nolen-Hoeksema, Wisco, \& Lyubomirsky, 2008) and dampening (i.e. the tendency to decrease positive emotional experiences) (Wood, Heimpel, \& Michela, 2003) have been related to increased and prolonged NA.

Similarly, the body of research on affective consequences employing EMA is also extensive. The EMA literature has confirmed previous findings regarding savoring (Jose, Lim, \& Bryant, 2012) and dampening (Li et al., 2017). However, contrasting results were observed for other strategies. For instance, cognitive reappraisal has been found to be related to increased PA (Richardson, 2017), which is consistent with previous research, but some studies failed to replicate its relationship with decreased NA (Brans et al., 2013; Brockman et al., 2017; Nezlek \& Kuppens, 2008; Pavani et al., 2016). Similarly, many EMA studies confirmed the association between suppression and subsequent increased NA and decreased PA (Brans et al., 2013; Brockman et al., 2017). Nevertheless, Richardson and colleagues observed no effects of suppression on NA (Richardson, 2017), and Heiy et al. found that suppression did not predict subsequent affect (Heiy \& Cheavens, 2014). Finally, the EMA literature confirmed the association between rumination and subsequent decreased PA and increased NA (Brans et al., 2013; Li et al., 2017; Pavani et al., 2016), albeit with mixed results (Genet \& Siemer, 2012; Heiy \& Cheavens, 2014).

\section{Physiological responses}

In addition to its influence on affective states, ER has also been shown to entail physiological concomitants. Thus far, rumination and its physiological effects have been widely investigated, showing that high rumination is associated with heightened activation of the hypothalamic-pituitary-adrenal axis (HPAA) and decreased heart rate variability (HRV) (Ottaviani et al., 2016). Moreover, suppression has been associated with increased sympathetic activation (Gross, 1998), whereas cognitive reappraisal has been linked to reduced physiological response (i.e. skin conductance) (Gruber, Hay, \& Gross, 2014). Finally, acceptance has been related to decreased electrodermal reactivity (Dunn, Billotti, Murphy, \& Dalgleish, 2009). 
A limitation of existent physiological research is that most studies have been conducted in laboratory settings with very few exceptions, such as the study by Brosschot and colleagues, which confirmed the association between rumination and lower HRV using a daily ecological experiment (Brosschot, Van Dijk, \& Thayer, 2007).

\section{Additional contributions of the EMA approach to the ER literature: Reciprocal interplay, moderation, and ER strategy use over time}

In addition to replicating traditional research, the implementation of EMA methodologies has shed light on important gaps in the previous ER literature, that would have been difficult to address with non-ecological research.

According to the EMA literature, not only can ER influence outcomes, but outcomes can also affect subsequent ER processes, thus closing the circle. Momentary affect and ER, for example, have been shown to reciprocally influence each other at a given point in time (Pavani et al., 2015); similarly, well-being has been shown to be both a predictor (Haines et al., 2016) and an outcome (Quoidbach, Berry, Hansenne, \& Mikolajczak, 2010) of ER. From a theoretical point of view, these findings provide further support for the need to ecologically and momentarily evaluate ER and related variables.

The use of EMA has also allowed for the exploration of moderators in the ER-to-outcomes relationship in reallife settings. The results so far suggest that the effects of ER on subsequent outcomes are not linear, but rather affected by contextual factors or by ER itself. In relation to the former, research has indicated that, on days when people experience fewer positive events, momentary savoring (Jose et al., 2012) and positive rumination (Li et al., 2017) are more relevant in increasing PA levels, whereas suppression is more strongly associated with subsequent lower PA on more stressful days (Richardson, 2017). Regarding the moderating role of ER strategies, a study found that rumination moderates the association between negative events and NA levels, so that unpleasant events predict higher NA when rumination use is high, but not when it is low (Genet \& Siemer, 2012).

Another contribution of the EMA approach is the exploration of the concurrent use of ER strategies over time. Heiy and colleagues showed that individuals use, on average, seven strategies to regulate each experienced emotion, which suggests that interactions among strategies may produce different outcomes (Heiy \& Cheavens, 2014). In this direction, McMahon and colleagues analyzed the concurrent use of reappraisal, 
suppression, and rumination, and they showed that reappraisal compensates for the negative effects of suppression and rumination on anxiety and depressive symptoms (McMahon \& Naragon-Gainey, 2018).

Finally, a key contribution of the EMA approach has been the exploration of the way ER strategy use is sequenced over time. Emerging evidence suggests that certain strategies are likely to affect subsequent strategy selection. For example, daily reappraisal has been shown to be associated with increased daily suppression (Brockman et al., 2017). Furthermore, ER strategies can affect subsequent antecedent-focused and responsefocused ER processes over time. An example of the way ER can affect subsequent situation selection comes from the study by Farmer and colleagues, showing that suppression of positive emotions leads to higher engagement in positive social events on the following days (Farmer \& Kashdan, 2012). Moreover, there is evidence suggesting that the adoption of certain strategies affects response modulation (i.e. the attempt to modify the experiential, physiological, or behavioral components of the emotional response). Weiss et al., for example, revealed that the adoption of distraction, cognitive reappraisal, and problem-solving predicts a reduction in marijuana consumption on the following days (Weiss, Bold, Sullivan, Armeli, \& Tennen, 2017). Likewise, the adoption of disengagement (e.g., denial, avoidance, wishful thinking, escape or inaction) and involuntary engagement strategies (e.g., rumination, impulsive or involuntary action) has been shown to lead to more problematic behaviors over time, such as lying or stealing (Silk, Steinberg, \& Morris, 2003).

\section{Conclusions and future directions}

Decades of experimental and observational research have resulted in a relatively comprehensive understanding of ER. Important milestones have already been achieved, such as identification of important antecedents (i.e., contextual and situational factors, affect, and personal goals) and consequences (i.e., affect and physiological states) associated with ER. Although the previous literature has clearly contributed to our understanding of ER, in this paper we argue that the implementation of EMA is fundamental in order to move the ER research forward. ER is a dynamic and complex process with elements that are likely to reciprocally interact with each other, so that antecedents eventually become consequences and vice-versa. Capturing such a complex process is challenging, especially when using laboratory experiments and retrospective ER measurements. Additionally, although laboratory studies are and will be important for ER and clearly facilitate the manipulation of a large number of elements in a controlled setting, the study of ER in real-life settings where 
multiple factors occur simultaneously is also necessary, because these scenarios are where people implement ER in their daily lives.

So far, the EMA literature has confirmed many of the findings achieved by laboratory experiments, again confirming that the current knowledge about ER is quite robust. Added to the existing literature, EMA research has provided important insights about the reciprocal nature of the relationship between ER and its antecedents and consequences, the potential role of ER and situational factors as moderators in the implementation of ER, and the key role of momentary ER in the study of subsequent ER implementation over time.

In sum, the implementation of EMA allows researchers to explore new dynamics of this process. However, this method could also make it possible to study ER through a multimodal approach that includes concurrent behavioral, physiological, and cognitive concomitants. Although interesting findings have already been achieved with this approach, further advances will require combining more objective and passive data collection methods with traditional EMA self-reports (Colombo, Fernandez Alvarez, Garcia-Palacios, et al., 2019). For example, behavioral information (i.e., sleep patterns, physical activity, social interactions, or social media use) can be obtained with smartphone embedded-sensors, and comfortable wrist-watches or wearable chest-straps can easily monitor and record physiological parameters in ecological settings, thus achieving a more comprehensive understanding of ER in daily life could be achieved (see Figure 1).

While acknowledging the aforementioned important contributions of EMA research on ER, this methodology is not without limitations. For instance, standardized and ad-hoc items to assess ER via EMA are currently not available, which makes it difficult to compare results across studies. Furthermore, this approach is more timeconsuming for participants (Colombo et al., 2018) and, because the data are collected in naturalistic environments, it becomes harder to have complete control over the setting (as opposed to laboratory studies). Considering the strengths and weaknesses of both methods, a combination of approaches should ideally be pursued in order to fully comprehend the complexity of ER. Several milestones have already been achieved, but the promising integration of different methods and technologies could provide a more comprehensive understanding of ER.

Clinical implications of a more ecological, momentary, and technology-assisted approach to ER are also worth mentioning. EMA could indeed change the way we assess ER in psychopathology by helping us to understand the dynamics of this process in different emotional disorders, as well as the impact of emotion dysregulation 
on patients' lives. In turn, this could help clinicians to assess patients' ER directly in daily life in order to recognize specific strategies that need to be targeted in the therapeutic process and provide therapeutic feedback to patients, which has been shown to be a valuable practice (Delgadillo et al., 2018). Using sensors to capture physiological responses or a person's behavior would further augment EMA's potential. The analysis of such multi-modal data can provide some insights into the interplay among emotions, physiology, and behavior, and systems that utilize these principles can enable the practical application of data-driven approaches. Particularly, machine learning algorithms are inherently attuned to interpreting complex interdependent information. Such algorithms have the advantage of continuously learning from observations, and they can be personalized for a specific user. This ambitious, technology-assisted implementation of EMA could, for instance, help us to identify person- or population-wide predictors of emotional states which, in turn, would foster the personalization of treatments and therapy plans.

\section{RECOMMENDATIONS FOR ADDITIONAL READING}

1. (Aldao, Sheppes, \& Gross, 2015): In this study, Aldao and colleagues discuss the relationship between ER, goals, and context, and they delve into the concept of ER flexibility, i.e. the ability to regulate emotions taking into account situational and contextual demands. Interestingly, specific recommendations to investigate this construct by means of EMA are provided.

2. (Gaggioli et al., 2013): Psychlog is an example of a mobile phone platform that can concurrently collect psychological (i.e. self-reports), physiological (HR monitoring), and activity (i.e. three-axial accelerometer) information in naturalistic settings.

3. (Ottaviani et al., 2015): Ottaviani and colleagues concurrently investigated the three dimensions of ER through an ecological approach. More specifically, cognitive, behavioral, and physiological correlates of rumination were explored in real-life for $24 \mathrm{~h}$, using a smartphone-based electronic diary and an ambulatory HR device.

4. (Blanke et al., 2019): Using data from four experience-sampling studies, Blanke and colleagues investigated the importance of the context on ER. According to their results, ER strategies are not adaptive or maladaptive; instead, adaptiveness is the result of the variability in choosing strategies based on situational demands. 


\section{FUNDING}

This work is supported by the Marie Curie EF-ST AffecTech Project, approved at call H2020 - MSCA - ITN - 2016 (project reference: 722022).

\section{REFERENCES}

Aldao, A. (2013). The Future of Emotion Regulation Research: Capturing Context. Perspectives on Psychological Science. https://doi.org/10.1177/1745691612459518

Aldao, A., Sheppes, G., \& Gross, J. J. (2015). Emotion Regulation Flexibility. Cognitive Therapy and Research, 39(3), 263-278. https://doi.org/10.1007/s10608-014-9662-4

Blanke, E. S., Brose, A., Kalokerinos, E. K., Erbas, Y., Riediger, M., \& Kuppens, P. (2019). Mix It to Fix It: Emotion Regulation Variability in Daily Life. Emotion. https://doi.org/10.1037/emo0000566

Brans, K., Koval, P., Verduyn, P., Lim, Y. L., \& Kuppens, P. (2013). The regulation of negative and positive affect in daily life. Emotion, 13(5), 926-939. https://doi.org/10.1037/a0032400

Brockman, R., Ciarrochi, J., Parker, P., \& Kashdan, T. (2017). Emotion regulation strategies in daily life: mindfulness, cognitive reappraisal and emotion suppression. Cognitive Behaviour Therapy, 46(2), 91113. https://doi.org/10.1080/16506073.2016.1218926

Brosschot, J. F., Van Dijk, E., \& Thayer, J. F. (2007). Daily worry is related to low heart rate variability during waking and the subsequent nocturnal sleep period. International Journal of Psychophysiology. https://doi.org/10.1016/j.ijpsycho.2006.07.016

Burns, A. B., Brown, J. S., Sachs-Ericsson, N., Ashby Plant, E., Thomas Curtis, J., Fredrickson, B. L., \& Joiner, T. E. (2008). Upward spirals of positive emotion and coping: Replication, extension, and initial exploration of neurochemical substrates. Personality and Individual Differences. https://doi.org/10.1016/j.paid.2007.08.015

Bylsma, L. M., \& Rottenberg, J. (2011). Uncovering the dynamics of emotion regulation and dysfunction in daily life with ecological momentary assessment. In Emotion Regulation and Well-Being. https://doi.org/10.1007/978-1-4419-6953-8_14

Catterson, A. D., Eldesouky, L., \& John, O. P. (2016). An experience sampling approach to emotion 
regulation: Situational suppression use and social hierarchy. Journal of Research in Personality. https://doi.org/10.1016/j.jrp.2016.04.004

Colombo, D., Cipresso, P., Fernández Alvarez, J., Garcia Palacios, A., Riva, G., \& Botella, C. (2018). An Overview of Factors Associated with Adherence and Dropout to Ecological Momentary Assessments in Depression. In B. K. Wiederhold, G. Riva, \& S. Bouchard (Eds.), Annual Review of CyberTherapy and Telemedicine (Vol. 16, pp. 11-17). Interactive Media Institute.

Colombo, D., Fernandez Alvarez, J., Garcia-Palacios, A., Cipresso, P., Botella, C., \& Riva, G. (2019). New Technologies for the Understanding, Assessment and Intervention of ER in Mental Health. Frontiers in Psychology. https://doi.org/10.3389/fpsyg.2019.01261

Colombo, D., Fernandez Alvarez, J., Palacios, A. G., Patané, A., Semonella, M., Cipresso, P., ... Botella, C. (2019). Current State and Future Directions of Technology- Based Ecological Momentary Assessment and Intervention for Major Depressive Disorder: A Systematic Review. Journal of Clinical Medicine, $8(4), 465$.

Delgadillo, J., de Jong, K., Lucock, M., Lutz, W., Rubel, J., Gilbody, S., ... McMillan, D. (2018). Feedbackinformed treatment versus usual psychological treatment for depression and anxiety: a multisite, openlabel, cluster randomised controlled trial. The Lancet Psychiatry. https://doi.org/10.1016/S22150366(18)30162-7

Eldesouky, L., \& English, T. (2018). Regulating for a reason: Emotion regulation goals are linked to spontaneous strategy use. Journal of Personality. https://doi.org/10.1111/jopy.12447

English, T., Lee, I. A., John, O. P., \& Gross, J. J. (2017). Emotion regulation strategy selection in daily life: The role of social context and goals. Motivation and Emotion, 41(2), 230-242. https://doi.org/10.1007/s11031-016-9597-z

Farmer, A. S., \& Kashdan, T. B. (2012). Social anxiety and emotion regulation in daily life: Spillover effects on positive and negative social events. Cognitive Behaviour Therapy. https://doi.org/10.1080/16506073.2012.666561

Fredrickson, B. L. (2001). The role of positive emotions in positive psychology. The broaden-and-build theory of positive emotions. The American Psychologist, 56(3), 218-226. https://doi.org/10.1037/0003066X.56.3.218 
Gaggioli, A., Pioggia, G., Tartarisco, G., Baldus, G., Corda, D., Cipresso, P., \& Riva, G. (2013). A mobile data collection platform for mental health research. Personal and Ubiquitous Computing, 17(2), 241251. https://doi.org/10.1007/s00779-011-0465-2

Genet, J. J., \& Siemer, M. (2012). Rumination moderates the effects of daily events on negative mood: Results from a diary study. Emotion. https://doi.org/10.1037/a0028070

Gross, J. J. (1998). Antecedent- and Emotion Regulation : Divergent Consequences Experience , Expression , Physiology. Journal of Personality and Social Psychology.

Gross, J. J. (2015). Emotion Regulation: Current Status and Future Prospects. Psychological Inquiry, 26(1), 1-26. https://doi.org/10.1080/1047840X.2014.940781

Gross, J. J., \& John, O. P. (2003). Individual Differences in Two Emotion Regulation Processes: Implications for Affect, Relationships, and Well-Being. Journal of Personality and Social Psychology. https://doi.org/10.1037/0022-3514.85.2.348

Gruber, J., Hay, A. C., \& Gross, J. J. (2014). Rethinking emotion: Cognitive reappraisal is an effective positive and negative emotion regulation strategy in bipolar disorder. Emotion. https://doi.org/10.1037/a0035249

Haines, S. J., Gleeson, J., Kuppens, P., Hollenstein, T., Ciarrochi, J., Labuschagne, I., ... Koval, P. (2016). The Wisdom to Know the Difference: Strategy-Situation Fit in Emotion Regulation in Daily Life Is Associated With Well-Being. Psychological Science. https://doi.org/10.1177/0956797616669086

Heiy, J. E., \& Cheavens, J. S. (2014). Back to basics: A naturalistic assessment of the experience and regulation of emotion. Emotion, 14(5), 878-891. https://doi.org/10.1037/a0037231

John, O. P., \& Gross, J. J. (2007). Individual Differences in Emotion Regulation. In J. J. Gross (Ed.), Handbook of Emotion Regulation (pp. 351-372). New York: The Guilford Press.

Jose, P. E., Lim, B. T., \& Bryant, F. B. (2012). Does savoring increase happiness? A daily diary study. Journal of Positive Psychology. https://doi.org/10.1080/17439760.2012.671345

Li, Y. I., Starr, L. R., \& Hershenberg, R. (2017). Responses to Positive Affect in Daily Life: Positive Rumination and Dampening Moderate the Association Between Daily Events and Depressive Symptoms. Journal of Psychopathology and Behavioral Assessment. https://doi.org/10.1007/s10862017-9593-y 
Marzano, L., Bardill, A., Fields, B., Herd, K., Veale, D., Grey, N., \& Moran, P. (2015). The application of mHealth to mental health: Opportunities and challenges. The Lancet Psychiatry. https://doi.org/10.1016/S2215-0366(15)00268-0

McMahon, T. P., \& Naragon-Gainey, K. (2018). The Moderating Effect of Maladaptive Emotion Regulation Strategies on Reappraisal: A Daily Diary Study. Cognitive Therapy and Research. https://doi.org/10.1007/s10608-018-9913-x

McRae, K., Heller, S. M., John, O. P., \& Gross, J. J. (2011). Context-Dependent Emotion Regulation: Suppression and Reappraisal at the Burning Man Festival. Basic and Applied Social Psychology. https://doi.org/10.1080/01973533.2011.614170

Nezlek, J. B., \& Kuppens, P. (2008). Regulating positive and negative emotions in daily life. Journal of Personality. https://doi.org/10.1111/j.1467-6494.2008.00496.x

Nolen-Hoeksema, S., Wisco, B. E., \& Lyubomirsky, S. (2008). Rethinking Rumination. Perspectives on Psychological Science, 3(5), 400-424. https://doi.org/10.1111/j.1745-6924.2008.00088.x

Ottaviani, C., Shahabi, L., Tarvainen, M., Cook, I., Abrams, M., \& Shapiro, D. (2015). Cognitive, behavioral, and autonomic correlates of mind wandering and perseverative cognition in major depression. Frontiers in Neuroscience. https://doi.org/10.3389/fnins.2014.00433

Ottaviani, C., Thayer, J. F., Verkuil, B., Lonigro, A., Medea, B., Couyoumdjian, A., \& Brosschot, J. F. (2016). Physiological concomitants of perseverative cognition: A systematic review and meta-analysis. Psychological Bulletin. https://doi.org/10.1037/bul0000036

Pavani, J. B., Le Vigouroux, S., Kop, J. L., Congard, A., \& Dauvier, B. (2016). Affect and Affect Regulation Strategies Reciprocally Influence Each Other in Daily Life: The Case of Positive Reappraisal, ProblemFocused Coping, Appreciation and Rumination. Journal of Happiness Studies. https://doi.org/10.1007/s10902-015-9686-9

Quoidbach, J., Berry, E. V., Hansenne, M., \& Mikolajczak, M. (2010). Positive emotion regulation and wellbeing: Comparing the impact of eight savoring and dampening strategies. Personality and Individual Differences. https://doi.org/10.1016/j.paid.2010.03.048

Richardson, C. M. E. (2017). Emotion regulation in the context of daily stress: Impact on daily affect. Personality and Individual Differences, 112, 150-156. https://doi.org/10.1016/j.paid.2017.02.058 
Sheppes, G., Scheibe, S., Suri, G., \& Gross, J. J. (2011). Emotion-regulation choice. Psychological Science. https://doi.org/10.1177/0956797611418350

Silk, J. S., Steinberg, L., \& Morris, A. S. (2003). Adolescents' Emotion Regulation in Daily Life: Links to Depressive Symptoms and Problem Behavior. Child Development, 74(6), 1869-1880. https://doi.org/10.1046/j.1467-8624.2003.00643.x

Srivastava, S., Tamir, M., McGonigal, K. M., John, O. P., \& Gross, J. J. (2009). The Social Costs of Emotional Suppression: A Prospective Study of the Transition to College. Journal of Personality and Social Psychology. https://doi.org/10.1037/a0014755

Suso-Ribera, C., Castilla, D., Zaragozá, I., Ribera-Canudas, M. V., Botella, C., \& García-Palacios, A. (2018). Validity, Reliability, Feasibility, and Usefulness of Pain Monitor, a Multidimensional Smartphone App for Daily Monitoring of Adults with Heterogeneous Chronic Pain. The Clinical Journal of Pain, 34(10), 1. https://doi.org/10.1097/AJP.0000000000000618

Troy, A. S., Shallcross, A. J., \& Mauss, I. B. (2013). A Person-by-Situation Approach to Emotion Regulation: Cognitive Reappraisal Can Either Help or Hurt, Depending on the Context. Psychological Science. https://doi.org/10.1177/0956797613496434

Weiss, N. H., Bold, K. W., Sullivan, T. P., Armeli, S., \& Tennen, H. (2017). Testing bidirectional associations among emotion regulation strategies and substance use: a daily diary study. Addiction. https://doi.org/10.1111/add.13698

Wood, J. V., Heimpel, S. A., \& Michela, J. L. (2003). Savoring Versus Dampening: Self-Esteem Differences in Regulating Positive Affect. Journal of Personality and Social Psychology. https://doi.org/10.1037/0022-3514.85.3.566 